\title{
Evaluation of the Manufacturability of "Shaft"-Type Parts with the Use of Complex Methods
}

\author{
Aleksey Sychugov ${ }^{1, a}$, Yulia Frantsuzova ${ }^{2, b^{*}}$ and Vladimir Salnikov ${ }^{2, c}$ \\ ${ }^{1}$ Tula State University, Department of Information security, Tula, Russia \\ 2 Tula State University, Department of Computer technology, Tula, Russia \\ axru2003@list.ru, bjulianna_1204@mail.ru, ‘vladimirsalnikov95@yandex.ru
}

Keywords: Adaptability, Labor Intensity, Unification, Design, Shaft

\begin{abstract}
This work is devoted to improving the quality of engineering design solutions in mechanical engineering on the basis of the comprehensive qualimetric evaluation of 'shaft'-type part that takes into account cost, complexity, design and technological unification of the parts.
\end{abstract}

\section{Introduction}

In today's competitive environment, product quality is the ultimate goal of any manufacturer and determines its value in the eyes of the consumer. A machine-building product designed to meet certain needs has properties that form its quality.

The development of a new product is a complex design task associated not only with achieving the required functional, but also technological level.

The process of ensuring the manufacturability of the product is contradictory and difficult to formalize, and during its implementation, conflicts of professional interests are inevitable between the developers of the design solution and its external and internal consumers. For the objective resolution of such conflicts, it is necessary to introduce a qualimetric assessment of the quality of the design solution according to the criterion of manufacturability, taking into account the following parameters: labor intensity, cost and unification of the design.

An important quality indicator is the degree of unification of the designed part both in relation to its actual design, and in relation to the possibility of using a unified standard technology for its manufacture. The implementation of an operational assessment of the processability of a design solution in the context of computer-aided design is possible in the form of an appropriate intelligent CAD subsystem.

Thus, the task of revealing the regularity of the manifestation of information, economic and organizational relations between the design, technological and metrological aspects of machinebuilding production in the qualimetric assessment of the manufacturability of the design of parts of the "shaft" type based on the construction of mathematical and information models of their condition and quality dynamics is relevant.

The set of product properties that determine the adaptability of its design to achieve optimal resource costs during production and operation to achieve the specified quality indicators is the manufacturability of the product design.

Ensuring the manufacturability of the product design at the design stage is a significant and at the same time the most difficult task of the designer. If the conformity of the machine design to a given functional purpose is easy to assess with objective numerical indicators, then such a complex and multifactorial concept as manufacturability is very difficult to quantify. The absence of generally accepted methods of numerical evaluation of technological efficiency does not allow to objectively resolve the inevitable conflicts of professional interests between the designer as a

(c) (1) Content from this work may be used under the terms of the Creative Commons Attribution 3.0 license. Any further distribution of this work must maintain attribution to the author(s) and the title of the work, journal citation and DOI. Published under license by Materials Research Forum LLC. 
supplier of design documentation (DD) and the technologist as its consumer.

It is obvious that each set of parameters of the part (shape and dimensions of surfaces, roughness, tolerances, design bases, the coefficient of workability of the material) corresponds to its own coefficient of manufacturability $N_{k}$. Criterion of adaptability of the part is exceeding the minimum permissible values $N_{\text {доп }}$, which is determined by technological possibilities of production, as a detail, quite technological for an advanced plant with modern equipment, can be unviable or unfeasible for a plant with outdated equipment.

\section{Mathematical modeling}

The number of processability indicators should be minimal, but sufficient to assess the processability.

The nomenclature of technological performance indicators and the method of their determination at each stage of CD development depends on the type of products and the type of production. Suggested list of indicators of technological items according to GOST Standard 14.201-83 "Integrating technology into product design" includes the complexity of manufacturing the product, the cost of technology and the coefficient of unification of the elements. The analysis of the research of Russian and foreign scientists in this subject area of science was carried out. The works of B. A. Yakimovich [10], V. I. Averchenkov [1], M. V. Novikova [5, 6], M. E. Popov [8] were studied. In these works, the complexity of manufacturing the product, the technological cost and the coefficient of unification of structural elements were considered.

The analysis of the above mentioned and works $[2,3,7,9]$ showed:

1. The authors did not consider the totality of all three indicators together;

2. To calculate the unification coefficient, only the applicability of structural elements in production and their standardization were taken into account, and the production capabilities of the enterprise and the unification of geometric dimensions were not taken into account. Its value is deterministic, either 1 or 0 .

Since labor intensity has a direct monetary value, it is usually included in the cost price. Therefore, the proposed assessment of manufacturability includes two main components: technological cost $C$ and unification, expressed by design $k_{d u}$ and technological $k_{t u}$ coefficients. Then

$$
N_{k}=\frac{2}{3}\|C\|+\frac{1}{3}\left(k_{d u}+k_{t u}\right)
$$

The weight coefficients $2 / 3$ and $1 / 3$ are introduced to reflect the fact that according to GOST Standard 14.201-83, cost, labor intensity and unification make an equal contribution to the manufacturability of the part.

Let's consider a mathematical model for determining the technological cost. The cost rate is calculated using the formula:

$$
\|C\|=\frac{C_{\text {nom, }}}{C_{T}}
$$

and is the ratio of the actual technological cost to the value $C_{n o m}$, that is either a technological cost of a detail-analog (previously manufactured item of similar size and design with 
a known cost), or nominal technology cost of a "simple" detail, for the calculation of which we take smooth shaft, dimensions of which coincide with the dimensions of considered details, and the surfaces have an average roughness $R a_{a v}$ :

$$
R a_{a v}=\sum_{i=1}^{n} \frac{R a_{i} \cdot l_{i}}{n \cdot l}
$$

where $R a_{j}$ - roughness of $j$-th treated surface; $l_{j}$ - length of $j$-th surface; $n$ - the number of treated surfaces; $l$ - overall shaft length.

The technological cost is calculated using the formula [4]:

$$
C_{\mathrm{T}}=C_{\mathrm{tot}}^{\mathrm{mat}}+T \cdot k_{\mathrm{mat}} \cdot\left(W_{\mathrm{w}}+C_{\mathrm{mh}}\right) \cdot\left(1+K^{\prime} / 100\right),
$$

and to calculate the labor intensity $T$, an empirical dependence [6] was used:

$$
T=M^{y} \cdot \sum_{i=1}^{n} S_{i}^{a} \cdot R a_{i}^{b} \cdot t_{i}^{c} \cdot k_{\text {мат }}
$$

where $C_{\text {tot }}^{\text {mat }}$ - the total cost of raw materials, RUB; $W_{\mathrm{w}}$ - the average hourly wage of the worker for the relevant production, rubles/hour; $C_{\mathrm{mh}}$ - cost of machine-hours RUB/hour; $K^{\prime}$ - average overhead costs, $\%$; $n_{-}$the number of design-technological elements (DTE); $k_{\text {mat }}$ - coefficient that takes into account the machinability of the material; $M$ - mass of the part, $\mathrm{kg}$; $S_{i_{-}}$the area of the shear layer of the i-th surface, $\mathrm{mm} 2 ; R a_{i}$ - the required roughness of the i-th surface, $\mu \mathrm{m} ; t_{-}$the tolerance of the $\mathrm{i}$-th surface, $\mathrm{mm} ; n_{-}$the number of the processed surfaces; $a, b, c, y_{-}$ empirical power indicators determined by the regression analysis method.

Next, we will consider the definition of the coefficients of design and technological unification of a body of rotation part type. The coefficient of design unification $k_{\mathrm{du}}$ is defined as

$$
k_{\mathrm{du}}=\frac{1}{n} \sum_{i=1}^{n} k_{\mathrm{du}_{\mathrm{i}}} \cdot \delta_{i} \text {, }
$$

where $n$ - the number of construction elements; $k_{\mathrm{Ky}_{\mathrm{i}}}$ - the coefficient of design unification of the i-th structural element of the part, depending on the shape and the value of the landing size (the size responsible for the accuracy of the location of the part in the assembly): for cylinder: 


$$
k_{\mathrm{du}_{\mathrm{i}}}=\left\{\begin{array}{l}
1, \text { if } D_{i}=1,6^{N}, \\
0,75, \text { if } D_{i}=1,25^{N}, \\
0,5, \text { if } D_{i}=1,12^{N}, \\
0,25, \text { if } D_{i}=1,06^{N}, \\
0, \text { if none of the conditions is met, }
\end{array}\right.
$$

for cone:

$$
k_{\mathrm{du}_{\mathrm{i}}}=\left\{\begin{array}{l}
1, \text { if } 2 \alpha_{i} \in A 1, \\
0,5, \text { if } 2 \alpha_{i} \in A 2, \\
0, \text { if none of the conditions is met, }
\end{array}\right.
$$

where $\alpha_{i}$ is the angle at the top of the cone; $A 1, A 2$ - the series of preferred angles according to GOST Standard 8593-81. The given constants $(1,6 ; 1,25 \ldots)$ express the series of preferred numbers for the cylinder diameter according to GOST Standard 8032-84.

For structural elements of other types (for example, shaped grooves, spherical surfaces), their presence in the catalog of analog parts is analyzed. If a similar element is found, then the coefficient $k_{\mathrm{du}_{\mathrm{i}}}=1$, otherwise $-k_{\mathrm{du}_{\mathrm{i}}}=0$.

In [4] it is indicated that from the point of view of manufacturability, it is necessary to observe a certain ratio between the dimensional tolerance on the surface and its roughness, due to the impossibility of achieving and measuring a rigid dimensional tolerance on an excessively rough surface. Therefore, for each surface to be treated, the following coefficient is introduced, taking into account the correctness of the roughness ratio and the tolerance field:

$$
\delta_{i}=\frac{1}{n} \sum_{j=1}^{n} \delta_{i j}
$$

where $\delta_{i j}=\left\{\begin{array}{l}0, \text { roughness exceeds } 5 \% \text { size tolerance, } \\ 1, \text { otherwise. }\end{array}\right.$

According to the data collected from the enterprises of the Tula region, it was revealed that one of the main reasons for the return of DD ( $45 \%$ of the number of returns at the stage of technological preparation) is the inconsistency of the basing schemes. In some cases, due to incorrect design bases, the tolerances for the closing dimensions turned out to be impossible, which required the re-processing of the DD. Therefore, the coefficient of technological unification of the part should primarily assess the degree of unity of the design and technological bases as the most important indicator of manufacturability. This coefficient cannot be calculated only on the basis of the data presented in the DD - it does not specify the technological bases. Therefore, according to the drawing, a lot of design bases (structural elements from which dimensions with tolerances are set) of the part $M_{\text {Кь }}$ are identified. Further, on the basis of a suitable typical technological process, a set of hypothetical technological bases $M_{\text {ть }}$ is determined. The coefficient $k_{\text {ту }}$ is the ratio of the 
number of matched bases $\left(k_{\text {сов }}\right)$ to the number of technological bases. $\left(k_{\text {ТБ }}\right)$ :

$$
k_{\mathrm{tu}}=\frac{k_{\mathrm{match}}}{k_{\mathrm{TB}}}
$$

When generating a hypothetical technological process, we should select typical equipment for each operation, if there is no additional information about the features of the equipment of a particular production. The equipment depends on the content of the operation itself, the overall dimensions of the workpiece, the properties of the material, the accuracy of the resulting size and the serial production. Next, you need to select a base scheme for each installation. For bodies of rotation, it depends mainly on the overall dimensions of the workpiece. By expert estimates were identified based on schema-based (in the cartridge, in the cartridge with the rest, in the Chuck with a steady rest and center, in the cartridge with the center, floating center, in centers, in centers with steady rests) from the length and diameter of the workpiece. The coordinates of the distribution center $\left(D_{\mathrm{L}}, L_{\mathrm{u}}\right)$ for the i-th base scheme will be calculated using the formulas:

$$
D_{\mathrm{c}_{i}}=\frac{1}{k_{i}} \sum_{j=1}^{k_{i}} d_{j}, L_{\mathrm{c}_{i}}=\frac{1}{k_{i}} \sum_{j=1}^{k_{i}} L_{j},
$$

where $i_{\text {- number of a schema-based; }} k_{i}$ - the number of parts defined by experts, for which the $\mathrm{i}$-th scheme-based is used; $d_{i}, l_{i}$ - diameter and length of the $\mathrm{i}$-th part.

The choice of the optimal layout $i_{\text {Oחт }}$ of the part with overall dimensions $d, l \mathrm{~d}$ is based on the condition of the minimum difference in the distance of points in the phase space $(D, L)$ for the selected material grade:

$$
i_{\mathrm{opt}}=\min _{i} \sqrt{\left(D_{\mathrm{c} i}-d\right)^{2}+\left(L_{\mathrm{c} i}-l\right)^{2}} .
$$

\section{Summary}

For each operation, we should choose a standard equipment. It depends on the content of the operation itself, the overall dimensions of the workpiece, the properties of the material, the accuracy of the resulting size and the serial production. Next, we need to solve the problem of building technological dimensional chains and checking them.

To check the adequacy of the model, a correlation coefficient was determined between expert estimates of the level of manufacturability of a number of parts of the type of bodies of rotation and estimates calculated according to the proposed method. The correlation coefficient was 0.84 , which indicates the adequacy of the mathematical model.

To effectively manage the processability at the design stage, it is not enough to simply evaluate it - we need to give recommendations to the designer, specifying the most non-technological parameters of the part.

The paper proposes a mathematical model of the state and dynamics of the quality of machine parts at the design stage, characterized by a qualimetric assessment of the manufacturability of their manufacture, taking into account the cost, labor intensity and technological unification of the 
elements of the part.

The developed method of qualimetric assessment of the manufacturability of design solutions and the optimal assignment of bases for parts of the "shaft" type, implemented in the future in the form of an intelligent CAD subsystem, allows us to increase the level of manufacturability by analyzing the information obtained during the development of the CD.

\section{References}

[1] Averchenkov V. I. Formalization of the construction and selection of progressive technologies that provide the required quality of products: Abstract of the dis. ... doc. tekhn. science: 05.02.08. - Bryansk, 1990.

[2] Akimov, I. V. Self-learning system for the rapid assessment of the complexity of manufacturing of machine parts: author. dis. cand. tekhn. science: 05.02.08. - Tula, 1999.

[3] I. V. Akimov, A. N. Inozemtsev, N. I. Pas'ko, Substantiation of the adaptive method for fast regulation of the complexity of the goods machine tools, Izvestiya TSU. Ser. Mashinostroenie. Tula, Issue 2 (1998) 216-221.

[4] Saati T. Decision-making: a method of analyzing hierarchies, Moscow, 1993.

[5] Amirov Yu. D Manufacturability of product design. Handbook, Moscow, 1990.

[6] Novikova M. V., Troitsky D. I. Integrated design and technological model of a part as a means of evaluating the labor intensity of its manufacture, Bulletin of Computer and Information Technologies, No. 1 (2006) 22-25.

[7] Volkhin K. A. Design documents and rules for their registration [Electronic resource]: textbook manual for students of technical sciences, Novosibirsk State Technical University. Novosibirsk, 2004. - Access mode: http://graph.power.nstu.ru/wolchin/umm/eskd/index.htm.

[8] Popov M. E. Automated methods of improving the manufacturability of product design in an integrated CAD/ M. E. Popov, A. M. Popov, Journal of mechanical engineering, No. 10 (2003) 48-53.

[9] Fominykh R. L. Development of automated subsystems the definition of constructivetechnological complexity, the complexity of the manufacture of parts and logistical level of multiproduct production: Author. dis. cand. tekhn. science: 05.13.06. - Izhevsk, 2003.

[10]. Yakimovich B. A., Kuznetsov A. P., Reshetnikov E. V. Model for calculating project costs for manufacturing machine-building products, Automation and Modern Technologies No. 5 (2003) 20-24. 